\title{
Production of a $\beta$-Glucosidase-Rich Cocktail from Talaromyces amestolkiae Using Raw Glycerol: Its Role for Lignocellulose Waste Valorization
}

\author{
Juan A. Méndez-Líter, Laura I. de Eugenio, Neumara L. S. Hakalin, Alicia Prieto *(D) and María Jesús Martínez *(D) \\ Centro de Investigaciones Biológicas Margarita Salas (CIB), Consejo Superior de Investigaciones \\ Científicas (CSIC), Ramiro de Maeztu 9, 28040 Madrid, Spain; jmendez@cib.csic.es (J.A.M.-L.); \\ lidem@cib.csic.es (L.I.d.E.); nhakalin@gmail.com (N.L.S.H.) \\ * Correspondence: aliprieto@cib.csic.es (A.P.); mjmartinez@cib.csic.es (M.J.M.)
}

check for

updates

Citation: Méndez-Líter, J.A.; de Eugenio, L.I.; Hakalin, N.L.S.; Prieto, A.; Martínez, M.J. Production of a $\beta$-Glucosidase-Rich Cocktail from Talaromyces amestolkiae Using Raw Glycerol: Its Role for Lignocellulose Waste Valorization. J. Fungi 2021, 7, 363. https://doi.org/ 10.3390/jof7050363

Academic Editor: Baojun Xu

Received: 14 April 2021

Accepted: 3 May 2021

Published: 6 May 2021

Publisher's Note: MDPI stays neutral with regard to jurisdictional claims in published maps and institutional affiliations.

Copyright: (c) 2021 by the authors. Licensee MDPI, Basel, Switzerland. This article is an open access article distributed under the terms and conditions of the Creative Commons Attribution (CC BY) license (https:/ / creativecommons.org/licenses/by/ $4.0 /)$.

\begin{abstract}
As $\beta$-glucosidases represent the major bottleneck for the industrial degradation of plant biomass, great efforts are being devoted to discover both novel and robust versions of these enzymes, as well as to develop efficient and inexpensive ways to produce them. In this work, raw glycerol from chemical production of biodiesel was tested as carbon source for the fungus Talaromyces amestolkiae with the aim of producing enzyme $\beta$-glucosidase-enriched cocktails. Approximately $11 \mathrm{U} / \mathrm{mL} \beta$ glucosidase was detected in these cultures, constituting the major cellulolytic activity. Proteomic analysis showed BGL-3 as the most abundant protein and the main $\beta$-glucosidase. This crude enzyme was successfully used to supplement a basal commercial cellulolytic cocktail (Celluclast 1.5 L) for saccharification of pretreated wheat straw, corroborating that even hardly exploitable industrial wastes, such as glycerol, can be used as secondary raw materials to produce valuable enzymatic preparations in a framework of the circular economy.
\end{abstract}

Keywords: biodiesel by-products; cellulases; fungi; hydrolases; wastes

\section{Introduction}

The current generation of wastes at worldwide level is a direct consequence of the inefficient use of natural resources in human activities. Industry has traditionally focused on the production of goods and services, consuming raw materials and generating wastes that remain in the environment. Valorization of the wide variety of mankind-produced waste has become an essential task for a sustainable world [1]. On the other hand, the need for energy and commodities is continuously increasing, while fossil fuels reserves are declining and finite. Therefore, it is imperative to learn how to use waste from other processes as secondary raw materials to produce alternative energy sources and materials.

Many carbon-rich industrial or urban residual streams are susceptible to re-valorization into value added products. In this sense, biodiesel is a renewable biofuel that can be produced from vegetal oils, animal fat, synthetic algae, or cooking oil waste [2]. In fact, fuels from renewable biomass are the cleanest sources of energy, having the potential to reduce the amount of $\mathrm{CO}_{2}$, nitrogen oxides (NOx), sulfur oxides (SOx), $\mathrm{CO}$, and other hazardous particles released to the atmosphere compared with fossil diesel [3]. One of the main problems derived from industrial production of biodiesel is the generation of huge amounts of glycerol as byproduct (10:1 ratio). The global biodiesel production has increased during the last decade, reaching more than $30.8 \mathrm{Mm}^{3}$ in 2016, and its production will achieve $41 \mathrm{Mm}^{3}$ in 2022 [2], which will be translated into millions of $\mathrm{m}^{3}$ of glycerol. Then, in the global market, glycerol has become an abundant raw material to be used, mainly in the chemical industry. However, the current industrial process for synthesis of biodiesel generates a by-product containing about 70-80\% glycerol together with water, catalysts, fatty acids, and salts. This makes the commercialization and valorization of this by-product 
difficult, since the glycerol required as raw material for pharmaceutical, cosmetic, and food industries requires high purity standards.

As the structural component of many lipids, glycerol is widely found in nature and some microorganisms can naturally use it to grow in the absence of additional carbon sources. These organisms can be potent tools for bioconversion of glycerol from biodiesel manufacturing into valuable microbial products, such as enzymes with industrial interest, medicinal drugs, antibiotics, and other chemicals [4-6]. Glycerol has substituted typical carbohydrates, such as sucrose or glucose, in industrial fermentations for production of 1,3-propanediol, dihydroxyacetone, succinic acid, propionic acid, citric acid, pigments, polyhydroxyalkanoates, biosurfactants, docosahexaenoic acid, lipids, or syngas [7].

On the other hand, lignocellulosic biomass can be converted into fermentable sugars by the synergistic action of a battery of enzymes including, exo- and endoglucanases and $\beta$-glucosidases, as well as other auxiliary enzymes [8]. Among them, $\beta$-glucosidases are essential to release free glucose, but commercial cellulase preparations, especially those from Trichoderma spp., are usually deficient in this activity and must be supplemented to increase their efficiency in saccharification processes. In this context, Talaromyces amestolkiae has been studied for its ability to secrete a powerful cellulosic cocktail with several $\beta$ glucosidases depending on the carbon source in the culture media [9]. Analysis of the secretomes released in those media revealed two mayor $\beta$-glucosidases. One of them, named as BGL-2, was induced exclusively by cellulosic substrates [10], while the other, BGL-3, was produced independently of culture carbon source [11]. BGL-3 is a versatile $1,4-\beta$-glucosidase that has proved to be very efficient for saccharification of wheat straw, but also highly active over $1,3-\beta$-glucose bonds [11].

In this work we propose the use of raw glycerol as an appropriate carbon source to produce efficient enzyme cocktails with high $\beta$-glucosidase levels using the fungus $T$. amestolkiae. The efficiency of these cocktails for degradation of lignocellulosic residues was evaluated, and plant biomass valorization is discussed in the framework of a circular economy approach.

\section{Materials and Methods}

\subsection{Chemicals, Microorganisms, Culture Media, and Crude Enzyme Extract Production}

Raw glycerol was obtained by separation of biodiesel after chemical transesterification of waste cooking oil and sodium metoxide [12]. It was directly supplied as carbon source in a basal medium for fungal growth. All other chemicals used in this work were of reagent grade and purchased from Sigma-Aldrich.

T. amestolkiae was isolated from cereal waste and included in the collection of the Institute Jaime Ferrán of Microbiology (IJFM) with the number A795. For spore production, the fungus was grown in potato dextrose agar (PDA, Difco) for 5 days at $28{ }^{\circ} \mathrm{C}$, and a suspension was obtained by placing $1 \mathrm{~cm}^{2}$ agar in $5 \mathrm{~mL}$ of a $1 \% \mathrm{NaCl}$ solution with $0.1 \%$ of Tween 80 . Pre-inocula were prepared by inoculating $200 \mu \mathrm{L}$ of this homogenous spore suspension in $250 \mathrm{~mL}$ flasks containing $50 \mathrm{~mL}$ of corn steep solid (CSS) medium (40 g/L glucose, $0.4 \mathrm{~g} / \mathrm{L} \mathrm{FeSO}{ }_{4} \cdot 7 \mathrm{H}_{2} \mathrm{O}, 9 \mathrm{~g} / \mathrm{L}\left(\mathrm{NH}_{4}\right)_{2} \mathrm{SO}_{4}, 4 \mathrm{~g} / \mathrm{L} \mathrm{K}_{2} \mathrm{HPO}_{4}, 26.3 \mathrm{~g} / \mathrm{L}$ corn steep solid, $7 \mathrm{~g} / \mathrm{L} \mathrm{CaCO}_{3}$, and $2.8 \mathrm{~mL} / \mathrm{L}$ soybean oil), previously autoclaved at $110{ }^{\circ} \mathrm{C}$ for $20 \mathrm{~min}$. The cultures were incubated at $28^{\circ} \mathrm{C}$ and $250 \mathrm{rpm}$ for 5 days.

Enzyme production was carried out in $1 \mathrm{~L}$ flasks containing $200 \mathrm{~mL}$ of Mandels minimal medium $\left(2.0 \mathrm{~g} / \mathrm{L} \mathrm{KH}_{2} \mathrm{PO}_{4}, 1.3 \mathrm{~g} / \mathrm{L}\left(\mathrm{NH}_{4}\right)_{2} \mathrm{SO}_{4}, 0.3 \mathrm{~g} / \mathrm{L}\right.$ urea, $0.3 \mathrm{~g} / \mathrm{L} \mathrm{MgSO}_{4} \cdot 7 \mathrm{H}_{2} \mathrm{O}$, $0.3 \mathrm{~g} / \mathrm{L} \mathrm{CaCl}_{2}, 5 \mathrm{mg} / \mathrm{L} \mathrm{FeSO}_{4} \cdot 7 \mathrm{H}_{2} \mathrm{O}, 1.6 \mathrm{mg} / \mathrm{L} \mathrm{MnSO}_{4} \cdot \mathrm{H} 2 \mathrm{O}, 1.4 \mathrm{mg} / \mathrm{L} \mathrm{ZnSO} 4 \cdot 7 \mathrm{H}_{2} \mathrm{O}$, and $1 \mathrm{~g} / \mathrm{L}$ Bacto peptone) [13], supplemented with $0.5 \%, 1 \%$, and $2 \%(w / v)$ raw glycerol as the carbon source, both unbuffered ( $\mathrm{pH}$ 4.5) and buffered with $100 \mathrm{mM}$ sodium phosphate $\mathrm{pH}$ 6. After autoclaving at $121^{\circ} \mathrm{C}$ for $20 \mathrm{~min}$, which was also useful for glycerol sterilization, the flasks were inoculated with $2 \mathrm{~mL}$ of the pre-inoculum, and incubated at $28{ }^{\circ} \mathrm{C}$ and $250 \mathrm{rpm}$ for 10 days, taking $1.5 \mathrm{~mL}$ aliquots daily for analytical determinations. After that time, cultures were filtered, concentrated, and dialyzed against a $\mathrm{pH} 4$ sodium acetate buffer at $10 \mathrm{mM}$ to produce the enzymatic cocktails. Concentration was achieved using an 
ultrafiltration cell (Amicon, Merck-Millipore, Kenilworth, NJ, USA) with a polysulfone membrane ( $3 \mathrm{kDa}$ cut-off, Millipore). The process was carried out with gentle stirring, using nitrogen gas for pressure (2 bar).

\subsection{Determination of Biomass, Proteins, Enzyme Activity, and Glycerol Consumption}

Aliquots from the cultures were centrifuged and filtered. The content of T. amestolkiae biomass in the different samples was determined from the dry weight of the lyophilized pellets. The other parameters were measured in the supernatants. Protein concentration was evaluated by using the commercial Bio-Rad Bradford assay (dye reagent concentrate) (Bio-Rad, CA, USA) and bovine serum albumin as the standard. $\beta$-glucosidase activity was tested following the hydrolysis of $0.1 \%(w / v) p$-nitrophenyl- $\beta$-D-glucopyranoside ( $p$ NPG, Sigma, St. Louis, MO, USA) at $50{ }^{\circ} \mathrm{C}$, in a $100 \mathrm{mM}$ sodium acetate buffer at $\mathrm{pH} 4$. The reaction was stirred at $1200 \mathrm{rpm}$ for $10 \mathrm{~min}$ in a Thermo shaker (T-100, SC-24 block), stopped by adding $2 \%(w / v)\left(1.42 \%\right.$ final concentration) of $\mathrm{Na}_{2} \mathrm{CO}_{3}$, and the $p$-nitrophenol $(p N P)$ released was spectrophotometrically measured at $410 \mathrm{~nm}\left(\varepsilon_{410}=15,200 \mathrm{M}^{-1} \mathrm{~cm}^{-1}\right)$. One unit of $\beta$-glucosidase activity was defined as the release of $1 \mu \mathrm{mol}$ of $p$ NP per minute. Avicelase (as indicative of exocellulase activity) and $\beta-1,4-$ endoglucanase activities were measured by determining the release of reducing sugars by the Somogyi-Nelson method, as previously described [9], with 1\% Avicel (Merck-Millipore) or 3\% low viscosity carboxymethylcellulose (Sigma), respectively, as substrates. One unit of activity was defined as the corresponding to the release of $1 \mu \mathrm{mol}$ of reducing sugar per minute. To determine glycerol consumption, the Glycerol Assay Kit (Sigma) was used according to the manufacturer's instructions.

All assays were performed in triplicate, and significant differences between samples were explored using Student's $t$-test, considering a $p$ value $<0.05$ as the limit for considering differences.

\subsection{Proteomic Analysis of T. amestolkiae Secretomes}

For proteomic analysis, samples from culture supernatants of T. amestolkiae grown in Mandels medium plus 1\% raw glycerol were analyzed. The process was carried out as previously described [9], with some minor variations. In brief, $2 \mathrm{~mL}$ of the liquid supernatants from 7-day-old cultures were centrifuged at 20,000 $\times g$ for $10 \mathrm{~min}$ to remove mycelium. Then, aliquots containing $5 \mu \mathrm{g}$ of proteins were first dissolved in SDS-PAGE loading buffer, denatured, loaded in a $12 \%$ SDS gel, and run for $10 \mathrm{~min}$, in order to remove non-protein compounds before the proteomic analysis. The whole protein band was excised from the gel, cut in small pieces, and digested with trypsin overnight at $37^{\circ} \mathrm{C}$. Then the peptides mixture was extracted and analyzed by nano-HPLC-MS/MS.

A NanoEasy HPLC (Proxeon Biosystems, Odense, Denmark), coupled to a nanoelectrospray ion source (Proxeon Biosystems), was used for all peptide separations. Samples were loaded onto a C18-A1 ASY-Column $2 \mathrm{~cm}$ precolumn (Thermo Scientific, MA, USA) and then eluted onto a Biosphere C18 column (C18, inner diameter $75 \mu \mathrm{m}, 15 \mathrm{~cm}$ long, $3 \mu \mathrm{m}$ particle size, Nano Separations, Nieuwkoop, Netherlands) as previously reported. Full-scan MS spectra $(m / z 300-1800)$ were acquired in the LTQ-Orbitrap Velos in the positive ion mode (Figure S1).

Mass spectra files were searched in a specific database against the T. amestolkiae genome. Precursor and fragments mass tolerance were set to $10 \mathrm{ppm}$ and $0.5 \mathrm{Da}$, respectively. Search parameters included a maximum of two missed cleavages allowed, carbamidomethylation of cysteines as a fixed modification, and oxidation of methionine as a variable modification. Peptides were validated through the algorithm Percolator [14], and only those with high and medium confidence were admitted (FDR 0.05). Protein identifications were only accepted if they contained at least two identified peptides. Results were inferred from data obtained from two technical replicates from two different biological samples. Relative quantification of the most abundant proteins in the samples analyzed was calculated from the number of peptide spectrum matches (PSMs) corresponding to each protein [15,16]. 


\subsection{Saccharification of Pretreated Wheat Straw}

The efficiency of T. amestolkiae enzyme cocktails as $\beta$-glucosidase supplements for saccharification was studied. For this, wheat straw subjected to three different pretreatments was used (Figure 1A): (i) acidic wheat straw (abbreviated as AcSE), obtained by steam explosion in the presence of dilute sulfuric acid (kindly provided by Abengoa Bioenergía, Babilafuente, Salamanca); (ii) neutral wheat straw (abbreviated as SE), obtained by steam explosion in the presence of water, also provided by Abengoa Bioenergía; (iii) and alkaline wheat straw (abbreviated as AP), prepared as follows: $5 \mathrm{~g}$ of wheat straw was incubated with $100 \mathrm{~mL}$ of $2 \% \mathrm{NaOH}$ for $40 \mathrm{~min}$ and $110^{\circ} \mathrm{C}$ using an autoclave, the solid material was then washed three times with water, the $\mathrm{pH}$ was adjusted to 5 with sulfuric acid, lyophilized, and stored. The composition of AP wheat straw was analyzed by sequential fractionation. In this process, the contents of cellulose, hemicellulose, and Klason lignin were determined through a quantitative acid hydrolysis [17]. In brief, the solid residue (300 mg) was treated for $1 \mathrm{~h}$ with $3 \mathrm{~mL}$ of $13.5 \mathrm{M}$ sulfuric acid $\left(\mathrm{H}_{2} \mathrm{SO}_{4}\right)$ at $30^{\circ} \mathrm{C}$ and $150 \mathrm{rpm}$. Then, $\mathrm{H}_{2} \mathrm{SO}_{4}$ was diluted to $0.5 \mathrm{M}$ and heated at $110^{\circ} \mathrm{C}$ for $1 \mathrm{~h}$. Sugars in the liquid fraction were identified and quantified by gas chromatography [18]. The slurry remaining after acid hydrolysis (Klason lignin) was washed until neutrality, oven dried at $55^{\circ} \mathrm{C}$, and weighted. All analyses were carried out in triplicate.

A

Wheat straw (WS) pretreatment
\begin{tabular}{|c|c|c|}
\hline \begin{tabular}{|c|} 
AcSE \\
Acid steam explosion \\
(with diluted $\mathrm{H}_{2} \mathrm{SO}_{4}$ )
\end{tabular} & $\begin{array}{c}\text { SE } \\
\text { Steam explosion } \\
\text { (with water) }\end{array}$ & $\begin{array}{c}\text { AP } \\
2 \% \mathrm{NaOH} \\
110^{\circ} \mathrm{C}, 40 \mathrm{~min}\end{array}$ \\
\hline
\end{tabular}

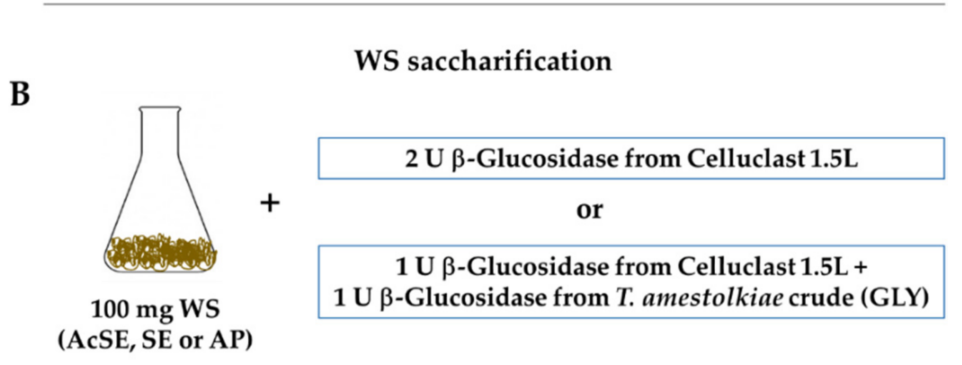

Figure 1. (A) Scheme of different wheat straw pretreatments used for enzymatic saccharification and (B) enzymatic doses used in the saccharification experiments.

For saccharification, $100 \mathrm{mg}$ of AcSE, SE, or AP wheat straw was incubated in $2 \mathrm{~mL}$ $100 \mathrm{mM}$ sodium acetate buffer at $\mathrm{pH} 4$ for $96 \mathrm{~h}$, at $40^{\circ} \mathrm{C}$ and $1200 \mathrm{rpm}$ in the presence of different enzymatic combinations (Figure 1B):

- $\quad 2 \mathrm{U}$ of $\beta$-glucosidase activity from Celluclast 1.5 L (Novozymes, Copenhagen, Denmark). This is a commercial cocktail, rich in cellobiohydrolase and endoglucanase activities, and was used for comparison purposes;

- $\quad 1 \mathrm{U}$ of $\beta$-glucosidase activity from Celluclast $1.5 \mathrm{~L}+1 \mathrm{U}$ of $\beta$-glucosidase activity from $T$. amestolkiae enzymatic crudes obtained in this work, using $1 \%$ glycerol as the carbon source.

\section{Results}

\subsection{BGL Production and Growth of T. amestolkiae in Media with Raw Glycerol}

The ascomycete T. amestolkiae has been proven as an excellent producer of robust and efficient cellulolytic enzymes in media with different carbon sources [9]. This finding suggested the convenience of testing cheaper carbon sources to obtain these cocktails, since they are rich in $\beta$-glucosidases, the key enzymes for cellulose saccharification. 
In this work, we studied the use of different concentrations of raw glycerol, an abundant waste generated from biodiesel production, to produce added-value enzyme cocktails. To analyze the potential of this carbon source, we followed the evolution of fungal growth and the extracellular levels of $\beta$-glucosidase over 10-day-old cultures $(240 \mathrm{~h})$. As observed in Figure 2A, T. amestolkiae cultures in unbuffered media reached maximal biomass values around $24 \mathrm{~h}$, regardless of the glycerol concentration assayed, although total biomass was higher as the glycerol concentration increased. The highest $\beta$-glucosidase levels were detected in cultures containing $1 \%$ or $2 \%$ glycerol (Figure $2 \mathrm{~B}$ ). BGL levels were low in the first $24-48 \mathrm{~h}$, and then they started to rise uninterruptedly at a high rate between $72 \mathrm{~h}$ and $168 \mathrm{~h}$. Surprisingly, both cultures produced around $8 \mathrm{U} / \mathrm{mL}$ at the final incubation time, despite that the fungal biomass was higher with $2 \%$ glycerol. Searching for an explanation to this result, it was observed that the $\mathrm{pH}$ was more acidic in the culture with $2 \%$ glycerol (pH 5) than in the other cultures ( $\mathrm{pH} 6-6.5)$. The acidification was detected at $168 \mathrm{~h}$ and maintained until the end of the incubation period.

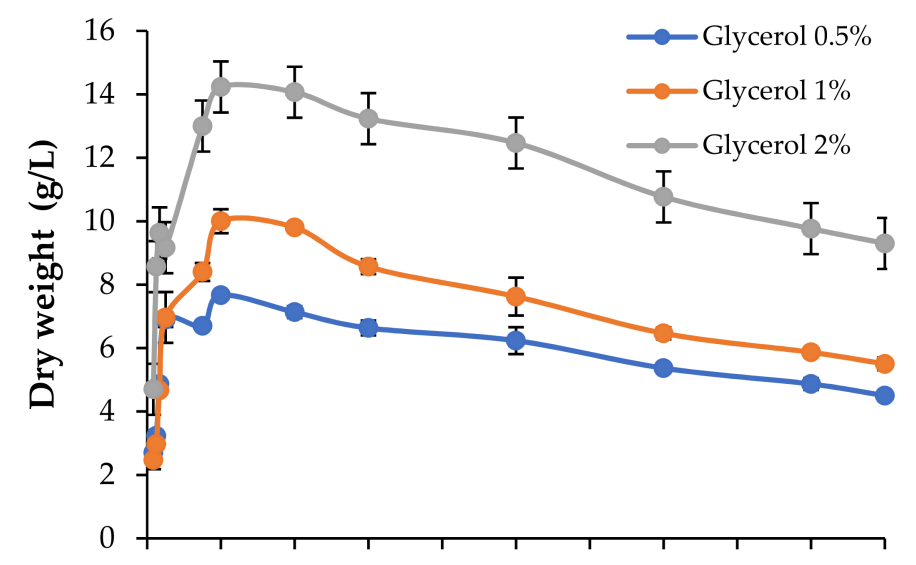

A

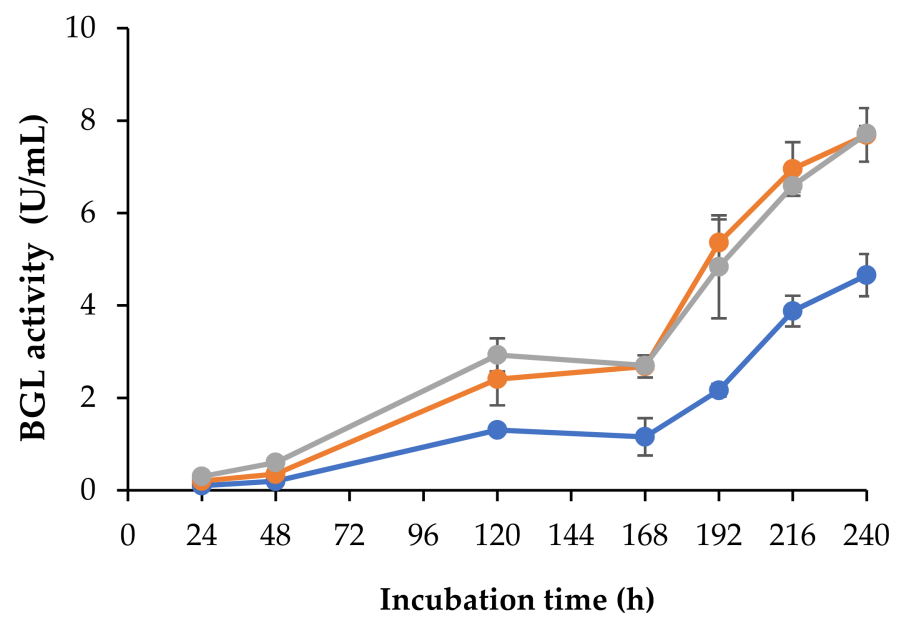

Figure 2. (A) Biomass and (B) $\beta$-glucosidase activity of T. amestolkiae growing in unbuffered cultures with raw glycerol $(0.5 \%, 1 \%$, and $2 \%)$, as the carbon source. All assays were performed in triplicate.

Thus, to investigate the effect of $\mathrm{pH}$ on BGL production, we repeated the experiment buffering all media with phosphate $\mathrm{pH} 6$ to maintain the $\mathrm{pH}$ as constant across the incubation period. In these conditions, BGL activity and fungal growth went in parallel, and the highest values for both were detected in cultures with $2 \%$ glycerol (Figure $3 \mathrm{~A}$ ). It should also be noticed that the total amount of secreted proteins detected in $2 \%$ glycerol unbuffered cultures was considerably lower than in the buffered ones $(0.10$ and $0.17 \mathrm{mg} / \mathrm{mL}$, respectively). These results suggest that $\mathrm{pH}$ should be strictly controlled in the cultures, since its value is related to the levels of secreted proteins. Buffered and unbuffered cultures 
with $0.5 \%$ and $1 \%$ glycerol followed a very similar behavior, where the $\mathrm{pH}$ was maintained in a $\mathrm{pH}$ range of 6-6.5.
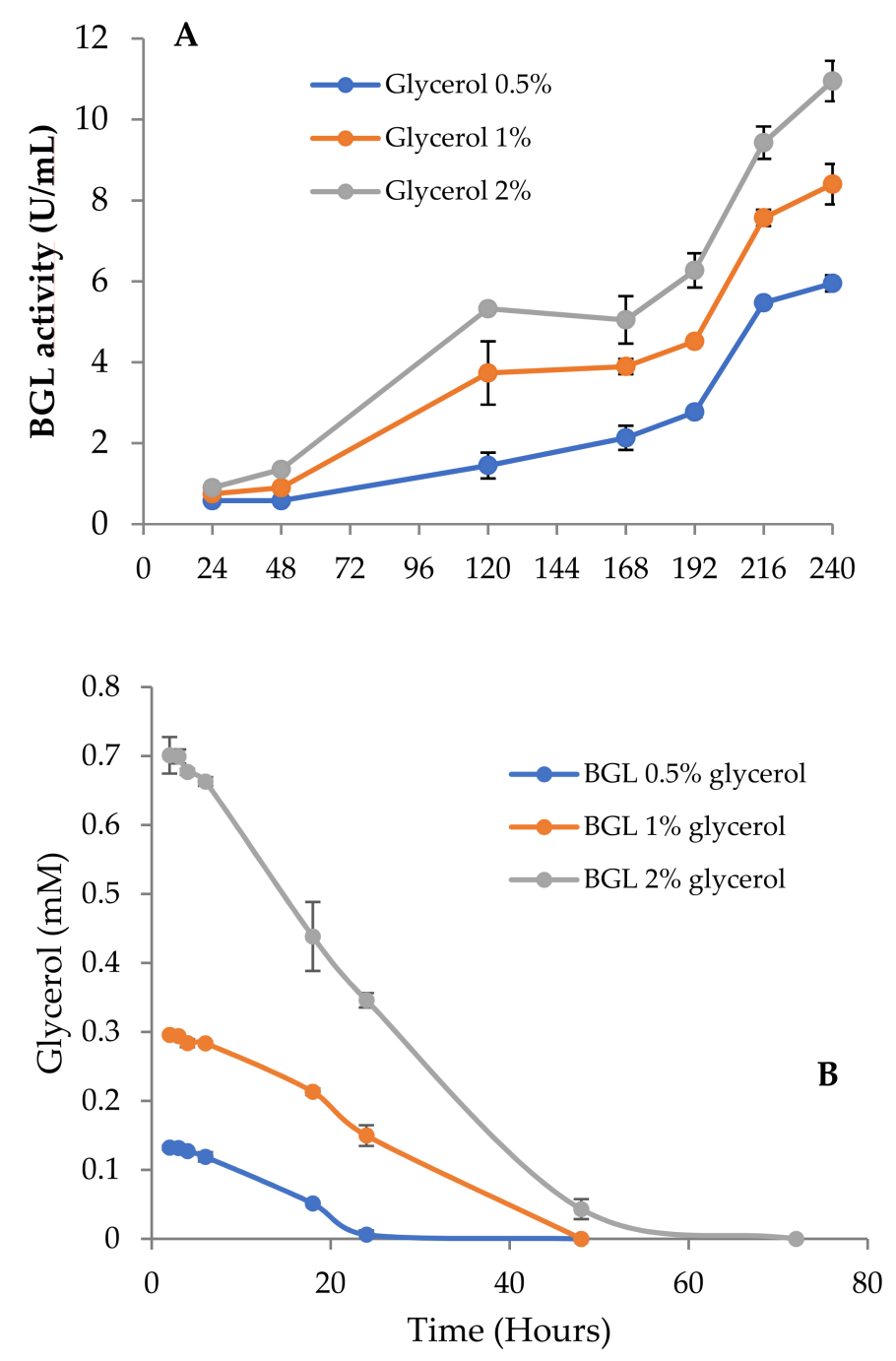

Figure 3. (A) $\beta$-glucosidase activity of T. amestolkiae growing in buffered cultures with raw glycerol $(0.5 \%, 1 \%$, and $2 \%)$, as the carbon source. (B) Glycerol consumption by T. amestolkiae in the buffered cultures. All assays were performed in triplicate.

On the other hand, these results confirm that T. amestolkiae does not require any specific inducer (cellulosic or non-cellulosic) to produce high amounts of $\beta$-glucosidases, as previously reported. This is probably due to the presence of BGL-3, an efficient $\beta$ glucosidase secreted under carbon starvation conditions [11]. BGL-3 is a $\beta-1,4$ glucosidase with a prominent activity on $\beta-1,3$ glucans. The high levels of this protein detected during secondary metabolism in the cultures may be due to its activity degrading the $\beta$-1,3-glucan released from the fungal cell wall during autolysis. As expected, the amount of other cellulolytic activities like avicelase or $\beta$-1,4-endoglucanase was negligible (data not shown), since these enzymes are usually released upon induction by cellulosic substrates.

Only a handful of studies have reported the production of fungal $\beta$-glucosidases using glycerol as the carbon source. For Penicillium echinulatum, glycerol was the preferred carbon source over cellulose, sugar cane bagasse (pretreated by steam explosion), or glucose [19]. Aspergillus niger NRRL 3112 could produce considerable amounts of this activity when grown on wheat bran and glycerol as co-substrates [20]. In other cases, the use of glycerol was not suitable for $\beta$-glucosidase production, as reported in Penicillium funiculosum [21]. Trichoderma reesei has been the most used fungus for producing cellulolytic 
cocktails, although it is well documented that the amount of $\beta$-glucosidase released by this fungus is insufficient for efficient hydrolysis of lignocellulosic biomass [22]. In this sense, one of the main advantages of the use of Aspergillus spp., Penicillium spp., or Talaromyces spp. lies in their higher secretion of $\beta$-glucosidase activity [22]. Many published reports allow us to compare its production among these fungal genera, mainly using crystalline cellulose as the carbon source. Different Aspergillus sp. were secreted between $0.5 \mathrm{U} / \mathrm{mL}$ and $2.5 \mathrm{U} / \mathrm{mL}$ of $\beta$-glucosidase $[23,24]$. Penicillium species, like Penicillium brasilianum or Penicillium decumbens, reached a total activity of $3.5 \mathrm{U} / \mathrm{mL}$ and $2.39 \mathrm{U} / \mathrm{mL}$, respectively $[25,26]$. Higher $\beta$-glucosidase levels, from 10 to $150 \mathrm{U} / \mathrm{mL}$, have been reported in strains of P. funiculosum, Penicillium occitanis, or Penicillium verriculosum $[21,27,28]$ using cellulosic inducers. It should be noted that the amounts of BGL observed in T. amestolkiae cultures in media with raw glycerol as the sole carbon source are among the highest reported to date. The fact that this high production was achieved using a by-product of the biodiesel industry makes it even more interesting.

Glycerol consumption was monitored and related with the increase of BGL activity (Figure 3B). In cultures with $0.5 \%$ glycerol, the depletion of the carbon source occurred in $24 \mathrm{~h}$, while in those with $1 \%$ and $2 \%$, it took around $48 \mathrm{~h}$. Low $\beta$-glucosidase levels were detected before glycerol exhaustion, but the activity started to rise slowly once consumed, and increased faster when carbon starvation conditions were fully established, expanding over time. The data in Figure 3B indicate that the maximal exploitation of glycerol for BGL production was obtained with $2 \%$ glycerol, so we chose this culture as the best model to produce BGL-rich cocktails.

The secretion of $\beta$-glucosidases in aged fungal cultures has been scarcely studied, although it would be interesting to know to what extent their production under carbon starvation is common among filamentous fungi. In the case of T. amestolkiae, it has been proved that induction of $\beta$-glucosidase BGL-3 was detected during carbon shortage. This enzyme is highly active on $\beta-1,3$ polysaccharides, suggesting its possible physiological role in cell wall metabolism during carbon starvation, releasing products that could be used as an alternative carbon source [11]. Some authors have reported the production of glycosyl hydrolases after carbon depletion in cultures of $A$. niger [29,30], but their finding was not discussed as a potential way to produce these enzymes. In any case, the versatility of $T$. amestolkiae to produce high levels of BGLs from different carbon sources, including some important industrial residues, should be exploited.

\subsection{Fungal Secretome Analysis}

An extracellular proteomic analysis of 7-day-old cultures was carried out to elucidate the protein profiles of $T$. amestolkiae cultures with $1 \%$ raw glycerol. The samples were subjected to tryptic digestion and LC-MS/MS of the whole peptide mixtures produced. The number of proteins identified was 148, similar to that reported in cultures with glucose or cellulosic substrates [9]. In the TIC scan of the proteomic analysis (Figure S1), an archive with al peptides was detected (Table S1), and a list with the 148 proteins detected (Table S2) can be found in the Supplementary Material Section of the article.

According to KOG, the functional role of most of the proteins identified in cultures with $1 \%$ raw glycerol was related to carbohydrate metabolism and transport (55.0\%), followed by enzymes involved in amino acid metabolism and transport (Table 1). These data also agree with those previously published for T. amestolkiae secretomes with glucose as the carbon source [9]. As the main objective of this work was to produce BGLs from contaminated glycerol waste, we started our analysis classifying the CAZymes (carbohydrate active enzymes), finding 80 out of 141 detected proteins (54\%). Among them, the most abundant were glycosyl hydrolases (GHs) from GH3, GH15, GH31, and GH55 families (Table 2). It is worth noting that GH15 enzymes were predominant in cultures with glucose as the carbon source [9], while in media with glycerol, GH3 proteins were the most abundant. The family GH3 is normally associated with enzymes degrading polysaccharides from lignocellulosic biomass, including many $\beta$-glucosidases, which supports the suitability of 
using this carbon source for $\beta$-glucosidase production. As expected, BGL-3 was found in the studied secretome (Table 3).

As already mentioned, this enzyme is secreted under carbon starvation [11], but other GHs potentially related with carbon depletion were also detected. This is the case of glucoamylases (GH15), $\alpha$-glucosidases (GH31), and exo- $\beta$-1,3-glucanases (GH55), related to the fungal autophagy process, since $1,3-\beta$-glucans and $1,4-\alpha$-glucan are components of the cell wall of many Talaromyces species [31]. Besides, it is noticeable that some of the most abundant proteins are related with the fungal autolysis processes. For example, cathepsin, which was detected in every condition, is a well-known protease that could be used by T. amestolkiae for degrading proteins and using its components as nutrients. The amino acids produced by cathepsin can be utilized by glutaminase, which has been related with glutamine metabolism under starvation conditions, when no other carbon source is present in the medium.

Table 1. Functional classification of the proteins identified in the secretome of 7-day-old T. amestolkiae cultures with raw glycerol as the carbon source, compared to those obtained in the same medium with glucose [9].

\begin{tabular}{ccc}
\hline & \multicolumn{2}{c}{ \% PSM } \\
\hline & Glycerol & Glucose \\
\hline A-RNA processing and modification & 0.63 & 0.22 \\
C-Energy production and conversion & 2.72 & 4.90 \\
E-Amino acid metabolism and transport & 13.11 & 10.38 \\
F-Nucleotide metabolism and transport & 0.74 & 1.42 \\
G-Carbohydrate metabolism and transport & 55.01 & 65.16 \\
I-Lipid metabolism & 0.09 & 0.17 \\
M-Cell wall/membrane/envelop biogenesis & 0.77 & 3.61 \\
O-Post-translational modification. protein turnover. & 1.96 & 1.71 \\
chaperone functions & 2.55 & 0.91 \\
Q-Secondary structure & 4.81 & 1.89 \\
S-General functional prediction only & 5.79 & 3.71 \\
T-Function unknown & 4.13 & 4.86 \\
\hline
\end{tabular}

Table 2. Glycosyl hydrolase families identified in secretomes from 7-day-old T. amestolkiae cultures growing in raw glycerol, compared to those obtained in the same medium with glucose as the carbon source [9].

\begin{tabular}{ccc}
\hline & & \% PSM \\
\hline GH Family & Glycerol & Glucose \\
\hline GH2 & 3.2 & 1.7 \\
GH3 & 16.6 & 16.3 \\
GH13 & 3.0 & 4.3 \\
GH15 & 10.1 & 28.4 \\
GH18 & 2.8 & 0.6 \\
GH20 & 3.7 & 4.8 \\
GH27 & 2.1 & 1.7 \\
GH31 & 8.6 & 11.8 \\
GH35 & 2.7 & 1.8 \\
GH55 & 8.1 & 3.9 \\
GH71 & 4.0 & 0.1 \\
GH72 & 3.2 & 1.3 \\
GH92 & 5.3 & 1.1 \\
GH127 & 3.6 & 3.2 \\
\hline
\end{tabular}


Table 3. Most abundant extracellular proteins identified in the T. amestolkiae secretome obtained from 7-day-old cultures growing in raw glycerol. BGL-3 is indicated in bold.

\begin{tabular}{|c|c|c|c|c|}
\hline Accession ID & \% PSM (Average) & $\begin{array}{l}\text { Predicted Protein } \\
\text { Function }\end{array}$ & Cazyme Family & $\begin{array}{c}M_{\mathrm{w}} \\
(\mathrm{kDa})\end{array}$ \\
\hline g377 (BGL-3) & 7.09 & beta-glucosidase & GH3 & 88.7 \\
\hline g3995 & 6.47 & Glutaminase & - & 76.4 \\
\hline g8295 & 3.59 & alpha-glucosidase & GH31 & 98.6 \\
\hline g2158 & 3.28 & Glucoamylase & GH15 & 65.2 \\
\hline g9324 & 3.18 & Exo-beta-1,3-glucanase & GH55 & 84.3 \\
\hline g2140 & 2.50 & Glucoamylase & GH15 & 67.7 \\
\hline g5915 & 2.23 & $\begin{array}{l}\text { non-reducing end } \beta \text {-L- } \\
\text { arabinofuranosidase }\end{array}$ & GH127 & 68.8 \\
\hline g4076 & 2.00 & $\begin{array}{l}\text { hexosaminidase } \\
\text { neutral/alkaline }\end{array}$ & GH20 & 67.9 \\
\hline g216 & 1.77 & $\begin{array}{c}\text { nonlysosomal } \\
\text { ceramidase }\end{array}$ & - & 160.0 \\
\hline g9148 & 1.58 & catalase & - & 79.1 \\
\hline
\end{tabular}

Regarding $\beta$-glucosidases, the most important result was the confirmation of the presence of BGL-3 as the most abundant extracellular protein. This is in good agreement with our previous results describing its production upon carbon exhaustion [9]. Besides, one GH1, recently characterized [32], and four GH3 $\beta$-glucosidases were also identified (Table 4). This profusion of $\beta$-glucosidases could contribute to explain why this activity was so high in this medium, as compared with other conditions

Table 4. Main hypothetical BGLs detected identified in the T. amestolkiae secretome obtained from 7-day-old cultures with raw glycerol as carbon source.

\begin{tabular}{cccc}
\hline Accession ID & \% PSM (Average) & Cazyme Family & $\boldsymbol{M}_{\mathbf{w}} \mathbf{( k D a )}$ \\
\hline g377 (BGL-3) & 7.09 & $\mathrm{GH} 3$ & 88.7 \\
g9150 & 1.54 & $\mathrm{GH} 3$ & 86.5 \\
g8384 & 0.85 & $\mathrm{GH} 1$ & 68.1 \\
g6857 & 0.79 & $\mathrm{GH} 3$ & 109 \\
g3139 & 0.30 & $\mathrm{GH} 3$ & 93.6 \\
g6753 & 0.09 & $\mathrm{GH} 3$ & 81.8 \\
\hline
\end{tabular}

In summary, the proteomic analysis of T. amestolkiae growing in raw glycerol confirmed the presence of BGL-3 and revealed that other interesting enzymes, including different $\beta$ glucosidases, can also be obtained for different biotechnological applications by exploiting the carbon starvation metabolism of T. amestolkiae.

\subsection{Wheat Straw Saccharification}

To check the efficiency of the novel enzyme cocktail obtained in this work for saccharification of lignocellulosic biomass, we used it as supplement of $\beta$-glucosidase activity for Celluclast 1.5 L (a basal cellulolytic preparation, deficient in this activity). Since lignocellulosic substrates have different characteristics, the most effective pretreatment and enzyme cocktail should be used, considering the properties of each raw material. Wheat straw is one of the most important agricultural residues worldwide. According to Iskalieva et al. [33], it is mainly composed of cellulose (38.8\%), hemicelluloses (39.5\%), and lignin (17.1\%). In the present work, three different pretreatments were used to make wheat straw cellulose more accessible: steam explosion (SE), steam explosion in the presence of dilute sulfuric acid (AcSE), and alkaline pretreatment (AP) (Figure 1). Steam explosion is one of the most commonly used methods for lignocellulose pretreatment, deconstructing and modifying part of the lignin, and solubilizing hemicellulose $[34,35]$. However, although cellulose is more accessible in the steam-exploded biomass, compounds derived from the partial 
hydrolysis of sugars and lignin remain embedded, producing adverse effects on downstream processes, including enzyme inhibition [36]. The use of dilute sulfuric acid could theoretically avoid this problem, since it helps to remove a bigger part of the lignin, but some of the polymer is always present in the material. On the other hand, the alkaline pretreatment with $\mathrm{NaOH}$ has been reported to increase cellulose digestibility by inducing a big reduction of the lignin content [37]. These pretreatments affect the composition of the material used for saccharification differently (Table 5).

Table 5. Main components of wheat straw pretreated by steam explosion in water (SE), steam explosion with dilute sulfuric acid (AcSE), or alkaline pretreatment (AP).

\begin{tabular}{cccc}
\hline & SE & AcSE & AP \\
\hline Cellulose & $49.0 \%$ & $43.6 \%$ & $71.8 \%$ \\
Hemicellulose & $15.4 \%$ & $17.1 \%$ & $24.1 \%$ \\
Lignin & $35.6 \%$ & $39.3 \%$ & $4.1 \%$ \\
\hline
\end{tabular}

In the case of alkaline pretreatment, most lignin was solubilized and removed, leaving a solid fraction enriched in polysaccharides. The two steam explosion treatments gave wheat straw slurries with different cellulose and hemicellulose contents. The AcSE treatment (dilute sulfuric acid) more strongly affected hemicellulose and lignin, forming compounds that produce adverse effects on downstream processes [38]. The results of the saccharification of pretreated wheat straw can be seen in Figure 4.

The combination of Celluclast $1.5 \mathrm{~L}$ with the $T$. amestolkiae cocktail improved the saccharification yield of steam-exploded wheat straw (with or without sulfuric acid), compared with Celluclast $1.5 \mathrm{~L}$ alone, reaching a cellulose conversion of $65 \%$ for SE and of $86 \%$ for AcSE. The commercial Celluclast $1.5 \mathrm{~L}$ contains an array of cellulolytic and hemicellulolytic activities in addition to BGL, which could theoretically improve saccharification. However, cellulose degradation with this cocktail was $41 \%$ for SE and 53\% for AcSE.

On the other hand, the saccharification of alkali-pretreated wheat straw was similar with both cocktails ( $86 \%$ for only Celluclast and $89 \%$ when supplemented with $\beta$ glucosidase from T. amestolkiae). One possible reason for this is the virtual lack of lignin in the alkali-pretreated wheat straw. It has been reported that lignin could trigger an irreversible cellulase inactivation, even when using pretreated lignocellulosic biomass, by two possible mechanisms: (i) forming a physical barrier that prevents enzyme access, and (ii) by non-productively binding of cellulolytic enzymes [34,39]. The non-productive binding of enzymes to lignin is recognized as a problem to overcome for improving saccharification efficiency of pretreated lignocellulosic biomass to fermentable sugars. A feasible explanation for our results is that the enzymes from the BGL-rich cocktails from T. amestolkiae were better than those contained in Celluclast $1.5 \mathrm{~L}$, avoiding the non-productive binding to lignin. This observation perfectly correlates with some studies that suggest that $\beta$ glucosidases and xylanases could avoid non-productive binding to lignin better than other cellulolytic enzymes [40]. Besides, it is remarkable that BGL-3, the most abundant cellulase in T. amestolkiae cocktails, possesses a fibronectin III domain (FnIII) [11]. Lima et al. [41], postulated that the FnIII domain strongly interacts with lignin fragments, preventing unproductive binding of cellulases to the lignin. Taking this into consideration, BGL-3 rich cocktails may be advantageous for the saccharification of pretreated residues where lignin is present, representing an effective and cheap alternative to current commercial enzymatic preparations. 


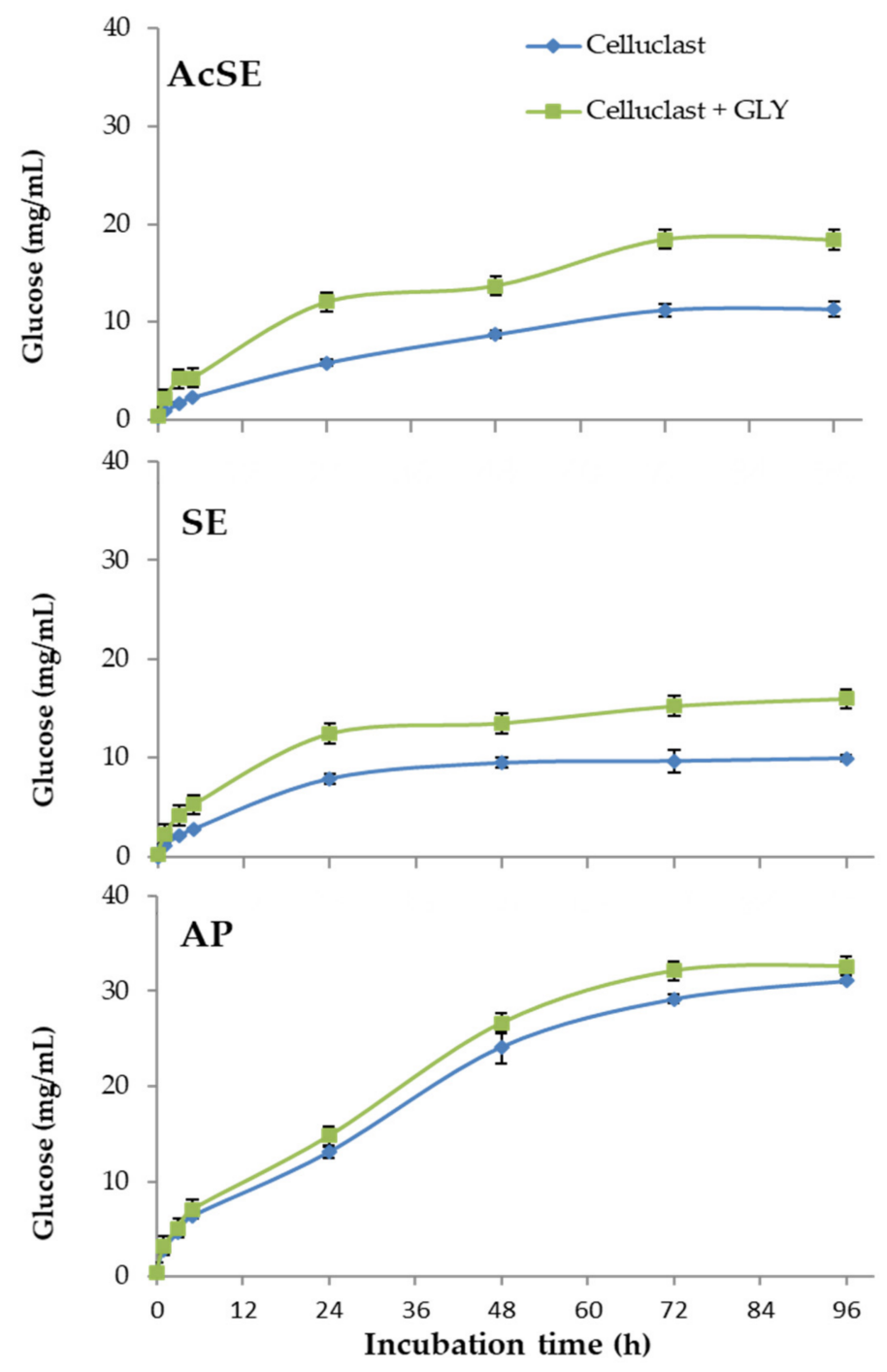

Figure 4. Saccharification of pretreated wheat straw (AcSE: acidic steam explosion; SE: steam explosion; AP: alkali pretreatment) with Celluclast $1.5 \mathrm{~L}$ (basal cocktail) and Celluclast 1.5 L supplemented with the T. amestolkiae cocktail obtained with raw glycerol as the carbon source (GLY). All assays were performed in triplicate.

\section{Conclusions}

In this work, we demonstrated the potential of the fungus T. amestolkiae for producing high levels of $\beta$-glucosidase activity using raw glycerol as the carbon source. Proteomic analysis of the crudes revealed that, although other minor glycosyl hydrolases were also found in the secretomes, BGL-3 was the main enzyme responsible for this behavior. This novel enzymatic cocktail was efficiently used to supplement the commercial cellulolytic preparation Celluclast $1.5 \mathrm{~L}$, increasing the glucose yield from wheat straw pretreated by steam explosion. This work set the foundations for expanding the industrial applications of T. amestolkiae and exploiting its efficient metabolism, able to use inexpensive carbon sources and to produce $\beta$-glucosidases and other value-added enzymes under carbon starvation.

Supplementary Materials: Supplementary materials are available online at https://www.mdpi. com/article/10.3390/jof7050363/s1.

Author Contributions: J.A.M.-L. and L.I.d.E. performed the experiments, the analysis of the results, and drafted the manuscript. N.L.S.H. performed the pretreatments of the wheat straw. M.J.M. and A.P. planned, reviewed, and edited the manuscript. All authors have read and agreed to the published version of the manuscript. 
Funding: This work has been funded by Projects GLYSUS RTI2018-093683-B-I00 (MCIU/AEI/FEDER), RETOPROSOST2 S2018/EMT-4459 (Comunidad de Madrid) and BioSFerA EU-H2020-NMBP-TR-IND.

Institutional Review Board Statement: Not applicable.

Informed Consent Statement: Not applicable.

Data Availability Statement: T. amestolkiae whole genome shotgun project is deposited at DDBJ/ ENA/GenBank under the accession MIKG00000000.

Acknowledgments: The authors thank the Proteomics and Genomics facility at CIB, Novozymes for providing the commercial cocktails, and Abengoa for supplying pretreated wheat straw. The authors acknowledge support of the publication fee by the CSIC Open Access Publication Support Initiative through its Unit of Information Resources for Research (URICI).

Conflicts of Interest: The authors declare that they have no competing interests.

\section{References}

1. Giusti, L. A review of waste management practices and their impact on human health. WASTE Manag. 2009, 29, 2227-2239. [CrossRef] [PubMed]

2. Monteiro, M.R.; Kugelmeier, C.L.; Pinheiro, R.S.; Batalha, M.O.; da Silva César, A. Glycerol from biodiesel production: Technological paths for sustainability. Renew. Sustain. Energy Rev. 2018, 88, 109-122. [CrossRef]

3. Lapinskiene, A.; Martinkus, P.; Rebzdaite, V. Eco-toxicological studies of diesel and biodiesel fuels in aerated soil. Environ. Pollut. 2006, 142, 432-437. [CrossRef] [PubMed]

4. Tang, S.; Boehme, L.; Lam, H.; Zhang, Z. Pichia pastoris fermentation for phytase production using crude glycerol from biodiesel production as the sole carbon source. Biochem. Eng. J. 2009, 43, 157-162. [CrossRef]

5. Luo, X.; Ge, X.; Cui, S.; Li, Y. Value-added processing of crude glycerol into chemicals and polymers. Bioresour. Technol. 2016, 215, 144-154. [CrossRef]

6. Magdouli, S.; Guedri, T.; Tarek, R.; Brar, S.K.; Blais, J.F. Valorization of raw glycerol and crustacean waste into value added products by Yarrowia lipolytica. Bioresour. Technol. 2017, 243, 57-68. [CrossRef]

7. Yang, F.; Hanna, M.A.; Sun, R. Value added use crude glycerol-a byproduct of biodiesel production. Biotechnol. Biofuels 2012, 5, $1-10$.

8. Guo, H.; Chang, Y.; Lee, D.J. Enzymatic saccharification of lignocellulosic biorefinery: Research focuses. Bioresour. Technol. 2018, 252, 198-215. [CrossRef]

9. de Eugenio, L.; Méndez-Líter, J.; Nieto-Domínguez, M.; Alonso, L.; Gil-Muñoz, J.; Barriuso, J.; Prieto, A.; Martínez, M. Differential $\beta$-glucosidase expression as a function of carbon source availability in Talaromyces amestolkiae: A genomic and proteomic approach. Biotechnol. Biofuels 2017, 10,1-14. [CrossRef]

10. Méndez-Líter, J.A.; Gil-Muñoz, J.; Nieto-Domínguez, M.; Barriuso, J.; De Eugenio, L.I.; Martínez, M.J. A novel, highly efficient $\beta$-glucosidase with a cellulose-binding domain: Characterization and properties of native and recombinant proteins. Biotechnol. Biofuels 2017, 10, 1-15. [CrossRef]

11. Méndez-Líter, J.A.; de Eugenio, L.I.; Prieto, A.; Martínez, M.J. The $\beta$-glucosidase secreted by Talaromyces amestolkiae under carbon starvation: A versatile catalyst for biofuel production from plant and algal biomass. Biotechnol. Biofuels 2018, 11, 1-14. [CrossRef]

12. de Sousa, M.H.; da Silva, A.S.F.; Correia, R.C.; Leite, N.P.; Bueno, C.E.G.; dos Santos Pinheiro, R.L.; de Santana, J.S.; da Silva, J.L.; Sales, A.T.; de Souza, C.C.; et al. Valorizing municipal organic waste to produce biodiesel, biogas, organic fertilizer, and value-added chemicals: An integrated biorefinery approach. Biomass Convers. Biorefinery 2021. [CrossRef]

13. Mandels, M.; Weber, J. The production of cellulases. In Cellulases and Their Applications; American Chemistry Society: Washington, DC, USA, 1969; pp. 391-414. ISBN 0-8412-0095-5.

14. Kall, L.; Krogh, A.; Sonnhammer, E.L.L. A combined transmembrane topology and signal peptide prediction method. J. Mol. Biol. 2004, 338, 1027-1036. [CrossRef]

15. Liu, H.B.; Sadygov, R.G.; Yates, J.R. A model for random sampling and estimation of relative protein abundance in shotgun proteomics. Anal. Chem. 2004, 76, 4193-4201. [CrossRef]

16. Schulze, W.X.; Usadel, B. Quantitation in Mass-Spectrometry-Based Proteomics. Annu. Rev. Plant Biol. 2010, 61, 491-516. [CrossRef]

17. Mardones, W.; Di Genova, A.; Cortés, M.P.; Travisany, D.; Maass, A.; Eyzaguirre, J. The genome sequence of the soft-rot fungus Penicillium purpurogenum reveals a high gene dosage for lignocellulolytic enzymes. Mycology 2018, 9, 59-69. [CrossRef]

18. Notararigo, S.; Nacher-Vazquez, M.; Ibarburu, I.; Laura Werning, M.; de Palencia, P.; Teresa Duenas, M.; Aznar, R.; Lopez, P.; Prieto, A. Comparative analysis of production and purification of homo- and hetero-polysaccharides produced by lactic acid bacteria. Carbohydr. Polym. 2013, 93, 57-64. [CrossRef] 
19. Schneider, W.D.H.; Goncalves, T.A.; Uchima, C.A.; Couger, M.B.; Prade, R.; Squina, F.M.; Pinheiro Dillon, A.J.; Camassola, M. Penicillium echinulatum secretome analysis reveals the fungi potential for degradation of lignocellulosic biomass. Biotechnol. Biofuels 2016, 9. [CrossRef]

20. Abdella, A.; Mazeed, T.E.-S.; El-Baz, A.F.; Yang, S.-T. Production of beta-glucosidase from wheat bran and glycerol by Aspergillus niger in stirred tank and rotating fibrous bed bioreactors. Process Biochem. 2016, 51, 1331-1337. [CrossRef]

21. Ramani, G.; Meera, B.; Vanitha, C.; Rao, M.; Gunasekaran, P. Production, Purification, and Characterization of a beta-Glucosidase of Penicillium funiculosum NCL1. Appl. Biochem. Biotechnol. 2012, 167, 959-972. [CrossRef]

22. Vaishnav, N.; Singh, A.; Adsul, M.; Dixit, P.; Sandhu, S.K.; Mathur, A.; Puri, S.K.; Singhania, R.R. Penicillium: The next emerging champion for cellulase production. Bioresour. Technol. Rep. 2018, 2, 131-140. [CrossRef]

23. Decker, C.H.; Visser, J.; Schreier, P. beta-glucosidases from five black Aspergillus species: Study of their physico-chemical and biocatalytic properties. J. Agicult. Food Chem. 2000, 48, 4929-4936. [CrossRef] [PubMed]

24. Riou, C.; Salmon, J.M.; Vallier, M.J.; Gunata, Z.; Barre, P. Purification, characterization, and substrate specificity of a novel highly glucose-tolerant beta-glucosidase from Aspergillus oryzae. Appl. Environ. Microbiol. 1998, 64, 3607-3614. [CrossRef] [PubMed]

25. Jorgensen, H.; Olsson, L. Production of cellulases by Penicillium brasilianum IBT 20888 - Effect of substrate on hydrolytic performance. Enzyme Microb. Technol. 2006, 38, 381-390. [CrossRef]

26. Sun, X.; Liu, Z.; Zheng, K.; Song, X.; Qu, Y. The composition of basal and induced cellulase system in Penicillium decumbens under induction or repression conditions. Enzym. Microb. Technol. 2008, 42, 560-567. [CrossRef]

27. Chaabouni, S.E.; Hadjtaieb, N.; Mosrati, R.; Ellouz, R. Preliminary assessment of Penicillium occitanis cellulase: A futher useful system. Enzyme Microb. Technol. 1994, 16, 538-542. [CrossRef]

28. Solov'eva, I.V.; Okunev, O.N.; Vel'kov, V.V.; Koshelev, A.V.; Bubnova, T.V.; Kondrat'eva, E.G.; Skomarovskii, A.A.; Sinitsyn, A.P. The selection and properties of Penicillium verruculosum mutants with enhanced production of cellulases and xylanases. Microbioloy 2005, 74, 141-146. [CrossRef]

29. Nitsche, B.M.; Jorgensen, T.R.; Akeroyd, M.; Meyer, V.; Ram, A.F.J. The carbon starvation response of Aspergillus niger during submerged cultivation: Insights from the transcriptome and secretome. BMC Genom. 2012, 13. [CrossRef]

30. van Munster, J.M.; Daly, P.; Delmas, S.; Pullan, S.T.; Blythe, M.J.; Malla, S.; Kokolski, M.; Noltorp, E.C.M.; Wennberg, K.; Fetherston, R.; et al. The role of carbon starvation in the induction of enzymes that degrade plant-derived carbohydrates in Aspergillus niger. Fungal Genet. Biol. 2014, 72, 34-47. [CrossRef]

31. Prieto, A.; Bernabe, M.; Leal, J.A. Isolation, purification and chemical characterization of alkali-extractable polysacharides from the cell-walls of Talaromyces species. Mycol. Res. 1995, 99, 69-75. [CrossRef]

32. Méndez-Líter, J.A.; Nieto-Domínguez, M.; Fernández De Toro, B.; González Santana, A.; Prieto, A.; Asensio, J.L.; Cañada, F.J.; De Eugenio, L.I.; Martínez, M.J. A glucotolerant $\beta$-glucosidase from the fungus Talaromyces amestolkiae and its conversion into a glycosynthase for glycosylation of phenolic compounds. Microb. Cell Fact. 2020, 19, 1-13. [CrossRef]

33. Iskalieva, A.; Yimmou, B.M.; Gogate, P.R.; Horvath, M.; Horvath, P.G.; Csoka, L. Cavitation assisted delignification of wheat straw: A review. Ultrason. Sonochem. 2012, 19, 984-993. [CrossRef]

34. Pan, X.J.; Xie, D.; Gilkes, N.; Gregg, D.J.; Saddler, J.N. Strategies to enhance the enzymatic hydrolysis of pretreated softwood with high residual lignin content. Appl. Biochem. Biotechnol. 2005, 121, 1069-1079. [CrossRef]

35. Galbe, M.; Wallberg, O. Pretreatment for biorefineries: A review of common methods for efficient utilisation of lignocellulosic materials. Biotechnol. Biofuels 2019, 12, 1-26. [CrossRef] [PubMed]

36. Garcia-Aparicio, M.; Ballesteros, I.; Gonzalez, A.; Oliva, J.; Ballesteros, M.; Negro, M. Effect of inhibitors released during steam-explosion pretreatment of barley straw on enzymatic hydrolysis. Appl. Biochem. Biotechnol. 2006, 129, 278-288. [CrossRef]

37. Kumar, R.; Wyman, C.E. Effects of cellulase and xylanase enzymes on the deconstruction of solids from pretreatment of poplar by leading technologies. Biotechnol. Prog. 2009, 25, 302-314. [CrossRef]

38. Moreno, A.D.; Ibarra, D.; Alvira, P.; Tomás-Pejó, E.; Ballesteros, M. A review of biological delignification and detoxification methods for lignocellulosic bioethanol production. Crit. Rev. Biotechnol. 2015, 35, 342-354. [CrossRef]

39. Gao, D.; Haarmeyer, C.; Balan, V.; Whitehead, T.A.; Dale, B.E.; Chundawat, S.P.S. Lignin triggers irreversible cellulase loss during pretreated lignocellulosic biomass saccharification. Biotechnol. Biofuels 2014, 7. [CrossRef]

40. Yarbrough, J.M.; Mittal, A.; Mansfield, E.; Taylor II, L.E.; Hobdey, S.E.; Sammond, D.W.; Bomble, Y.J.; Crowley, M.F.; Decker, S.R.; Himmel, M.E.; et al. New perspective on glycoside hydrolase binding to lignin from pretreated corn stover. Biotechnol. Biofuels 2015, 8. [CrossRef]

41. Lima, M.A.; Oliveira-Neto, M.; Kadowaki, M.A.S.; Rosseto, F.R.; Prates, E.T.; Squina, F.M.; Leme, A.F.P.; Skaf, M.S.; Polikarpov, I. Aspergillus niger beta-Glucosidase Has a cellulase-like tadpole molecular shape: Insights into gycoside hydrolase famyly 3 (GH3) beta-glucosidase structure and function. J. Biol. Chem. 2013, 288, 32991-33005. [CrossRef] 